\begin{tabular}{ll|l}
\cline { 2 - 3 } Intervent Neurol 2017;6:25-30 & $\begin{array}{l}\text { (c) 2016 S. Karger AG, Basel } \\
\text { www.karger.com/ine }\end{array}$ \\
$\begin{array}{l}\text { DoI: 10.1159/000452137 } \\
\text { Published online: October 19, } 2016\end{array}$ & \\
\hline
\end{tabular}

\title{
Endovascular Therapy Demonstrates Benefit over Intravenous Recombinant Tissue Plasminogen Activator Based on Repeatedly Measured National Institutes of Health Stroke Scale
}

\author{
Liqiong Fan $^{\mathrm{a}}$ Sharon D. Yeatts ${ }^{\mathrm{a}}$ Lydia D. Foster ${ }^{\mathrm{a}}$ Pooja Khatri ${ }^{\mathrm{b}}$ \\ Thomas Tomsick $^{c}$ Joseph P. Broderick ${ }^{b}$ Yuko Y. Palesch ${ }^{a}$

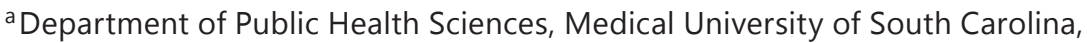 \\ Charleston, SC, and Departments of ${ }^{\mathrm{b}}$ Neurology and ${ }^{\mathrm{C}}$ Radiology, University of \\ Cincinnati, Cincinnati, OH, USA
}

\section{Keywords}

Endovascular treatment - Ischemic stroke - Longitudinal cohort study · National Institutes of Health Stroke Scale

\begin{abstract}
Background and Purpose: The Interventional Management of Stroke (IMS) III trial was a randomized controlled trial designed to compare the effect of endovascular therapy after intravenous recombinant tissue plasminogen activator (i.v. rt-PA) as compared to i.v. rt-PA alone. The primary outcome was modified Rankin Scale at 90 days. Secondary outcomes included National Institutes of Health Stroke Scale (NIHSS), which was assessed repeatedly through 90 days. The objective of this analysis is to evaluate the treatment effect of endovascular therapy over time on NIHSS. Methods: 656 subjects were enrolled in the IMS III trial, including 434 subjects randomized to endovascular therapy and 222 to i.v. rt-PA only. NIHSS scores evaluated at $40 \mathrm{~min}, 24 \mathrm{~h}$, Day 5, and Day 90 were included in the analysis. A covariance structure model was used to investigate the treatment effect on NIHSS over time, adjusting for relevant covariates including baseline stroke severity. Model assumptions were valid. Results: Based on the covariance structure model, after adjusting for relevant baseline covariates, a significant time-by-treatment interaction effect $(p=0.0137)$ was observed. Only NIHSS at Day 90 showed a significant treatment effect $(p=0.0473)$, with subjects in the endovascular arm having a lower NIHSS (less neurologic deficit) compared to the i.v. rt-PA arm. Conclusions: The
\end{abstract}


Fan et al.: Analysis of NIHSS Repeated Measurements

IMS III trial demonstrated an endovascular treatment effect based on the secondary outcome of NIHSS. However, the magnitude of this treatment effect varied by the time of assessment. It was only at Day 90 that the endovascular arm had a significantly lower NIHSS compared to that in the i.v. rt-PA arm.

(C) 2016 S. Karger AG, Basel

\section{Introduction}

The Interventional Management of Stroke (IMS) III trial was a phase III, randomized, controlled, multicenter trial designed to evaluate the efficacy of endovascular therapy (EVT) following intravenous recombinant tissue plasminogen activator (i.v. rt-PA) compared to i.v. rt-PA alone. Briefly, subjects had moderate-to-severe stroke, defined based on a baseline National Institutes of Health Stroke Scale (NIHSS) of 10 or more or an NIHSS of 8-9 with documentation of large artery occlusion on CT angiography, and were treated with i.v. rt-PA within $3 \mathrm{~h}$ of onset. After i.v. rt-PA, subjects were randomized with 2:1 allocation to EVT versus i.v. rt-PA. The primary outcome was the modified Rankin Scale evaluated at 90 days after onset. Detailed study information regarding the trial methodology and primary results has previously been published $[1,2]$. The trial was terminated early when the prespecified futility boundary was crossed after 656 out of 900 subjects had been enrolled.

The NIHSS, which has been shown to predict outcome well after stroke [3-8], was a prespecified secondary outcome of the trial. In addition to the baseline measurement used to define severity, the NIHSS evaluation was repeated at 40 min after i.v. rt-PA initiation, $24 \mathrm{~h}$, 5 and 90 days. Therefore, we assessed the benefit of EVT using the NIHSS outcome measure and explored the temporal pattern of the treatment effect.

\section{Methods}

NIHSS measured at 40 min after i.v. rt-PA initiation, 24 h, 5 and 90 days were used as outcomes. Because the distribution of NIHSS at each individual time point is skewed, the treatment effect at each time point was evaluated first independently using the Wilcoxon Rank Sum test. The correlation across time points was assessed via the Spearman Correlation coefficient. Then, a covariance structure model was applied to investigate the treatment effect over time, adjusting for prognostic covariates. The residual plots of the model were assessed and the normality assumption was met. All subjects enrolled in the IMS III trial were included for the repeated measure analysis. The covariance structure model takes into account the correlation across repeated measurements by estimating the covariance matrix pattern while making inference. Interaction between time of the NIHSS measurement and treatment was also included. The analysis model can be illustrated as

NIHSS $=\operatorname{Tr} t+$ Time $+($ Time $\times \operatorname{Tr} t)+\gamma X+\varepsilon$

where $X$ is a vector of covariates including age, time from symptom onset to treatment, and baseline stroke severity defined according to the dichotomized baseline NIHSS (NIHSS $\leq 19$ vs. NIHSS $\geq 20$, to be consistent with the primary analysis [1]). All covariates were prespecified in the statistical analysis plan. The normality assumption of residuals was checked and met.

The analysis was also explored in the subgroup of subjects with identified occlusion on baseline CT angiography, given the trend towards treatment effect on the primary outcome in this subgroup [9], and the similar selection paradigm in recent positive trials $[9,10-12]$.

All tests were conducted at a two-sided $\alpha$ level of 0.05 . All analyses were carried out using SAS $^{\circledR}$ version 9.4. 
Fig. 1. Distribution of NIHSS at each time point. $p$ values presented are results comparing the treatment groups independently at each time point. EVT, endovascular therapy; i.v. rt-PA, intravenous recombinant tissue plasminogen activator; NIHSS, National Institutes of Health Stroke Scale.

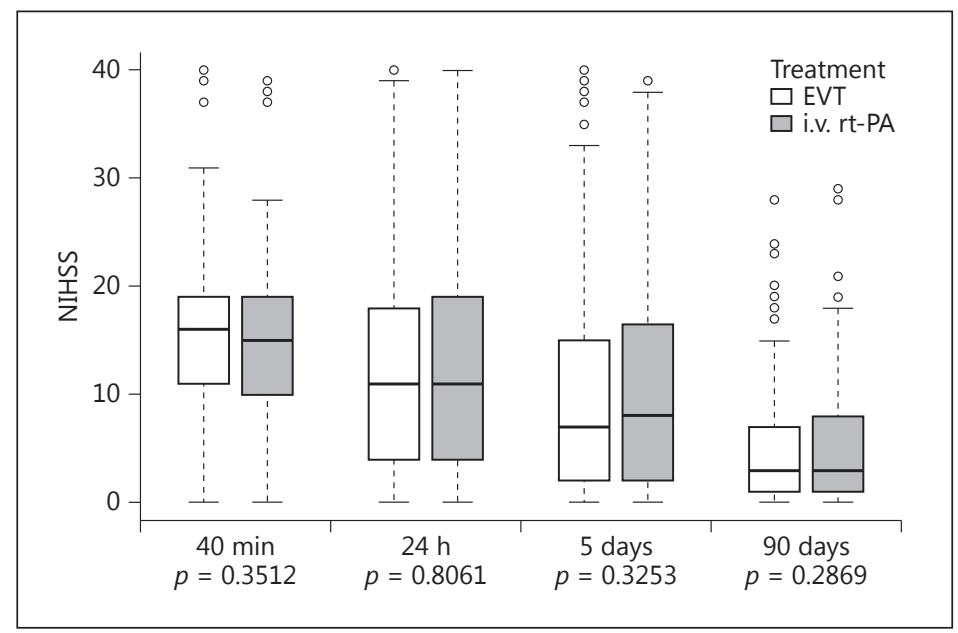

Table 1. Results of the covariance structure model for the fixed effects

\begin{tabular}{lc}
\hline Predictors & $p$ \\
\hline Treatment (EVT vs. i.v. rt-PA) $^{\mathrm{a}}$ & 0.6580 \\
Time $^{\mathrm{a}}$ & $<0.0001$ \\
EVT time $^{\mathrm{a}}$ & 0.0137 \\
Baseline stroke severity (moderate vs. severe) $<0.0001$ \\
Age, years & 0.0013 \\
Symptom onset to i.v. rt-PA, min & 0.0450 \\
\hline \multicolumn{2}{c}{ EVT, endovascular therapy; i.v. rt-PA, intravenous recombinant } \\
tissue plasminogen activator. a Time of National Institutes of Health \\
Stroke Scale assessment (from i.v. rt-PA initiation): 40 min, 24 h, 5 and \\
90 days.
\end{tabular}

\section{Results}

A total of 656 subjects were included in the analysis with 434 in the treatment (EVT) group and 222 in the control (i.v. t-PA only) group. Figure 1 presents the distribution of the observed NIHSS by treatment group at each time point, as well as the results of the Wilcoxon Rank Sum test ( $p$ values) comparing the treatment groups independently at each time point. There was insufficient evidence to declare a treatment effect at any individual time point.

Pairwise correlation of the NIHSS between each time point is observed. In general, all time points are at least moderately correlated, with the highest correlation observed between $24 \mathrm{~h}$ and day $5(r=0.88)$ and the lowest between $40 \mathrm{~min}$ and day $90(r=0.46)$. The association wanes over time. However, no obvious covariance structure pattern was identified based on the correlation coefficients. Therefore, an unstructured pattern was adopted and tested for the subsequent covariance structure model analysis.

Table 1 presents the results of the covariance structure model. The interaction between the treatment and time is statistically significant, which indicates that the effect of EVT on the NIHSS varies over time, i.e., the treatment effect on the NIHSS varies according to the evaluation time point. Therefore, care must be taken when interpreting the main effects. Age, time from symptom onset to treatment, and stroke severity at baseline are all important prognostic covariates in the model. Figure 2 present the results of the estimated NIHSS based on 
Fig. 2. The estimated NIHSS after accounting for the correlation among the repeated measurements. EVT, endovascular therapy; i.v. rt-PA, intravenous recombinant tissue plasminogen activator; NIHSS, National Institutes of Health Stroke Scale.

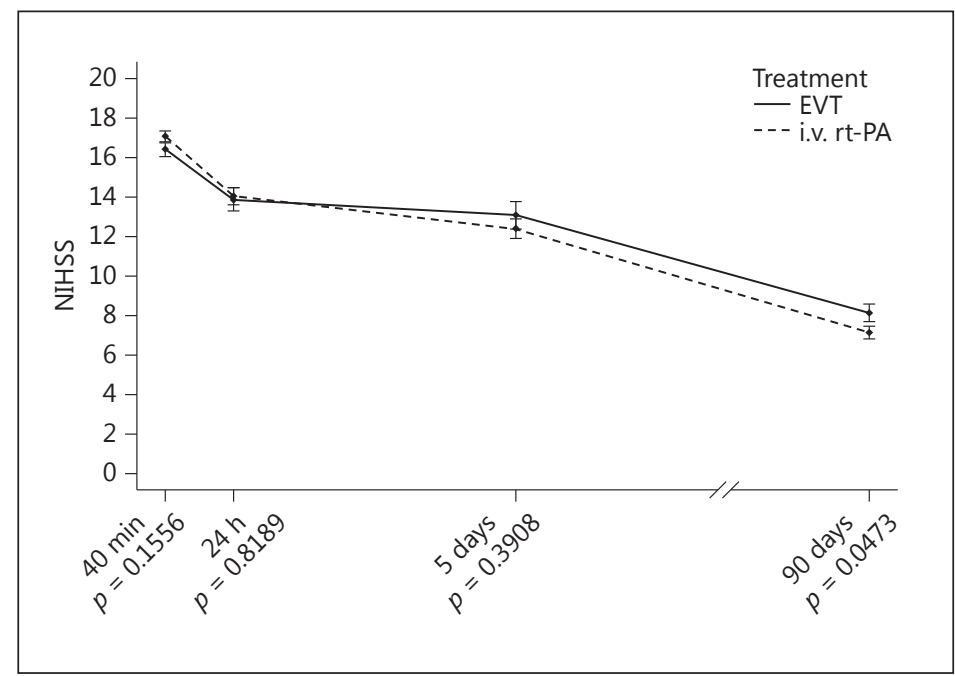

the covariance structure model, which models the repeated measurements simultaneously. After accounting for the correlation among the repeated measurements, a significant treatment effect is observed at Day $90(p=0.0473)$. The estimated effect of EVT on the NIHSS based on the covariance structure model is 1 unit decreasing compared to that of the i.v. rt-PA therapy only.

Among the subgroup with occlusion on baseline CT angiography $(n=282)$, a similar trend can be seen, with a slightly larger estimated treatment effect at 90 days $(-1.45,95 \% \mathrm{CI}$ : $[-3.13,0.24])$.

\section{Discussion}

The IMS III trial demonstrated a modest treatment effect in favor of EVT based on the secondary outcome of NIHSS using a repeated analysis approach. However, the magnitude of this treatment effect varied by the time of assessment. Only at Day 90 did the endovascular arm have a significantly lower NIHSS than the IV-tPA arm. It is clear that time is an important factor in terms of evaluating the benefit of reperfusion therapies including EVT. At least 3 months are needed to maximize potential effects of treatment. In addition, the magnitude of the difference in NIHSS found in this analysis was small and maybe of debatable clinical significance and potentially within the range difference between rates [13-15]. However, this difference was found significant irrespective of the variability among raters. Meanwhile, this diminished treatment effect may not be surprising in the setting of the older generation devices and inclusion of patients without proven arterial occlusions prior to enrollment. We also conducted the laterality analysis, as one of the reviewers recommended, based on the location of strokes (right vs. left hemisphere, results are not shown). Stroke laterality was not significantly associated with the outcome $(p=0.4133)$ in the analytic model, and the final model remained the same. Although the primary analysis of the IMS III trial was not able to demonstrate the efficacy of EVT in the primary outcome measure, this secondary analysis provides a hint of such effect in an unselected IMS III population and is consistent with subsequent subgroup analyses in IMS III subjects with a large artery occlusion upon baseline CT angiography and those subjects with an NIHSS $\geq 20$ at 6-12 months. Our findings of possible benefit of EVT in the IMS III trial are also consistent with subsequent 5 large, multi- 
center, randomized trials $[11,12,16,17]$ that have now incontrovertibly demonstrated the benefit of EVT in patients selected on the basis of imaging demonstration of large vessel occlusion.

Our analysis approach - covariance structure modeling - takes into account the correlation between the measurements. It reduces the variability among the measurements and therefore increases the power for detecting the potential treatment effect [18]. In addition, the covariance structure model can handle data with missing at random when the covariance structure is estimated, which is an assumption we make for our data. This type of longitudinal analysis is not a common primary analysis in randomized controlled trials, especially in stroke. However, analysis of longitudinal data can improve power and reduce sample size and should be considered for the design of future studies.

There are some limitations of the analysis. First of all, the primary outcome (modified Rankin Scale 0-2 at 90 days) of the IMS III trial was not significantly different between the 2 treatment arms; this secondary analysis, although prespecified, cannot be considered conclusive evidence of efficacy. The evaluation at $40 \mathrm{~min}$ and $24 \mathrm{~h}$ may not be blinded to the treatment assignment, which may introduce some bias to the results. Multiplicity should also be taken into consideration while interpreting the significance of the results. Finally, the IMS III trial included earlier generation devices for EVT and did not select patients based on the presence of baseline occlusion. This may have introduced greater heterogeneity to the data and diluted the treatment effect, thereby contributing to the futility finding of the overall trial.

\section{Acknowledgements}

This work is supported by NIH/NINDS: PI: Joseph P. Broderick, MD; Co-PI: Thomas Tomsick, MD; University of Cincinnati Clinical Coordinating and Angographic Imaging Center; U01-Ns052220; PI: Yuko Palesch, PhD; Statistics and Data Coordinating Center; Medical University of South Carolina; U01-NS054630; ClinicalTrials.gov Identifier: NCT 00359424.

\section{Disclosure Statement}

The authors declare no potential conflicts of interest with respect to the research, authorship, and/or publication of this article.

\section{References}

1 Broderick JP, et al: Endovascular therapy after intravenous t-PA versus t-PA alone for stroke. N Engl J Med 2013;368:893-903.

2 Khatri P, et al: Methodology of the Interventional Management of Stroke III Trial. Int J Stroke 2008;3:130-137.

3 Adams HP Jr, et al: Baseline NIH Stroke Scale score strongly predicts outcome after stroke: a report of the Trial of Org 10172 in Acute Stroke Treatment (TOAST). Neurology 1999;53:126-131.

4 De Haan R, et al: A comparison of five stroke scales with measures of disability, handicap, and quality of life. Stroke 1993;24:1178-1181.

5 Frankel MR, et al: Predicting prognosis after stroke: a placebo group analysis from the National Institute of Neurological Disorders and Stroke rt-PA Stroke Trial. Neurology 2000;55:952-959.

6 Glymour MM, et al: Lesion characteristics, NIH stroke scale, and functional recovery after stroke. Am J Phys Med Rehabil 2007;86:725-733.

7 Harrison JK, McArthur KS, Quinn TJ: Assessment scales in stroke: clinimetric and clinical considerations. Clin Interv Aging 2013;8:201-211.

8 Muir KW, et al: Comparison of neurological scales and scoring systems for acute stroke prognosis. Stroke 1996;27:1817-1820. 
Fan et al.: Analysis of NIHSS Repeated Measurements

9 Demchuk AM, et al: Recanalization and clinical outcome of occlusion sites at baseline CT angiography in the Interventional Management of Stroke III trial. Radiology 2014;273:202-210.

10 Campbell BC, et al: Endovascular therapy for ischemic stroke with perfusion-imaging selection. N Engl J Med 2015;372:1009-1018.

11 Goyal M, et al: Randomized assessment of rapid endovascular treatment of ischemic stroke. N Engl J Med 2015; 372:1019-1030..

12 Jovin TG, et al: Thrombectomy within 8 hours after symptom onset in ischemic stroke. N Engl J Med 2015;372: 2296-2306..

13 Dewey HM, et al: Interrater reliability of the National Institutes of Health Stroke Scale: rating by neurologists and nurses in a community-based stroke incidence study. Cerebrovasc Dis 1999;9:323-327.

14 Katz B, et al: Interrater reliability of the National Institutes of Health Stroke Scale: rating by emergency room physicians and neurologists in a population-based study (P5.140). Neurology 2015;84(suppl 14):P5.140.

15 Goldstein LB, Samsa GP: Reliability of the National Institutes of Health Stroke Scale. Extension to non-neurologists in the context of a clinical trial. Stroke 1997;28:307-310

16 Berkhemer OA, et al: A randomized trial of intraarterial treatment for acute ischemic Stroke. N Engl J Med 2015;372:11-20.

17 Saver JL, et al: Stent-retriever thrombectomy after intravenous t-PA vs. t-PA alone in stroke. N Engl J Med 2015;372:2285-2295.

18 Littell RC, Pendergast J, Natarajan R: Modelling covariance structure in the analysis of repeated measures data. Stat Med 2000;19:1793-1819. 